\title{
Padrões de Simetrias e Recursão em Ladrilhos Hidráulicos e Bandeiras: Exercícios Didáticos e Construção de Conhecimento Sobre Patrimônio Histórico
}

\author{
Symmetry and Recursion Patterns in Hydraulic Tiles and Fanlights: Didactic Exercises and Building \\ Knowledge about Historic Heritage
}

\author{
Janice de Freitas Pires \\ Universidade Federal de Pelotas, Brasil \\ janicefpires@hotmail.com \\ Carolina Machado Alves \\ Universidade Federal de Pelotas, Brasil \\ carolinamachadoalves@hotmail.com \\ Luisa dalla Vecchia \\ Universidade Federal de Pelotas, Brasil \\ luisafelixd@gmail.com
}

\author{
Adriane Borda Almeida da Silva \\ Universidade Federal de Pelotas, Brasil \\ adribord@hotmail.com \\ Ana Paula Dametto \\ Universidade Federal de Pelotas, Brasil \\ anapauladametto@hotmail.com
}

\begin{abstract}
This paper describes activities of analysis and composition of patterns based on architectural elements of buildings of historic heritage. During two separate workshops, which used a method previously established, the rules of symmetry and recursion were recognized in the geometry of these architectural elements and new patters were created through dynamic processes of digital representation. Such activities can stimulate the students' capacity of recognizing design actions, contributing to their perception of the design problems and, thus, to the knowledge for design practice.
\end{abstract}

Keywords: Symmetry and recursion patterns; Historic heritage; Architectural elements; Digital graphic representation.

\section{Introdução}

Os casarões de Pelotas do entorno da Praça Coronel Pedro Osório configuram o maior conjunto arquitetônico neo-renascentista preservado na América Latina. Este conjunto é reconhecido pelo IPHAN, órgão de caráter nacional, o qual promoveu o tombamento de quatro exemplares. Estes casarões possuem vários componentes arquitetônicos de interesse patrimonial tais como pisos feitos com a arte milenar dos mosaicos e artefatos metálicos.

Os mosaicos possuem composição decorativa em duas ou mais cores, formada de peças regulares ou irregulares, com dimensões variadas. Encontram-se em pedra natural, terracota, cerâmica, massa vidrosa ou mármore, fixados sobre superfície estável (OLIVEIRA, 1994; LÉON, 1998). Vestíbulos, calçadas, salas, avarandados, cozinhas trazem a marca da Indústria dos mosaicos da cidade, que chegou a possuir 17 fábricas. Este tipo de indústria se estabeleceu a partir de 1914, mas hoje existe uma única fábrica remanescente deste passado: a Fábrica de Mosaicos de Pelotas (http://fabricademosaicos.com.br/ladrilho-hidraulico-fabrica-deladrilhos-pelotas). Ela se utiliza praticamente dos mesmos padrões de mosaicos utilizados desde o seu início. Padrões art nouveau, art déco, florais e geométricos (LEÓN, 1998). Segundo Oliveira et al (1994), o ladrilho hidráulico (ou, para LEÓN, 1998, mosaico hidráulico), é constituído de cimento e areia e trabalhado em formas e prensas. Esta autora destaca que os moldes são grelhas de ferro fundido, que apresentam variados padrões e estilos de desenho, o que possibilita diferentes composições.

Os artefatos metálicos compõem sacadas, guarda-corpos, balcões, esquadrias, bandeiras entre outros. As bandeiras, em especial, foram muito utilizadas para favorecer a iluminação e ventilação dos interiores, principalmente em edificações do século XIX. Apareciam sobre as portas e as janelas e podiam ser fixas ou móveis (ASCIS, 2007).

Em trabalho anterior (Borda et al, 2012), um dos casarões de Pelotas, reconhecido como o de número 2, foi selecionado como objeto de estudo para promover análises de padrões compositivos dos ladrilhos hidráulicos ali presentes. As análises foram realizadas sob o conceito de simetrias de friso (SANZ E MORATALLA, 1999). A partir da identificação das regras compositivas de simetria envolvidas, estruturou-se uma metodologia de análise e 
construíram-se materiais didáticos com o uso de recursos informáticos. Estes materiais incluíam exercícios compositivos a partir de processos estáticos, semidinâmicos e dinâmicos (CELANI, 2003).

Especificamente para o estudo dos mosaicos utilizaram-se processos informáticos dinâmicos, automatizando cada uma das composições por simetrias. Estes processos informáticos utilizamse do recurso de visualização de janelas do AUTOCAD (CELANI, 2003). A partir da configuração destas janelas com diferentes pontos de vista e níveis de aproximação, é possível visualizar o desenho de um padrão sem aplicar transformações geométricas ao elemento fundamental. Estes exercícios foram planejados para serem inseridos em disciplinas de geometria de estágios iniciais de formação em arquitetura. Isto para que os estudantes já passem a reconhecer as potencialidades dos meios digitais para auxiliar processos criativos. O método proposto possibilita gerar diferentes composições: a partir da mudança de regra de simetria mantendo-se o elemento base da composição ou a partir da mudança do elemento base e mantendo-se as regras.

Através desse tipo de atividade buscou-se atribuir significado ao estudo da geometria, promovendo-se a compreensão de que os processos compositivos dos elementos em questão fundamentamse essencialmente no conceito de simetria. E, principalmente, contribuir à valorização do patrimônio arquitetônico e seu potencial para alimentar processos criativos no projeto de arquitetura.

O presente trabalho resulta deste mesmo propósito de promover uma postura criativa frente ao repertório extraído de um patrimônio relevante. Tem-se estruturado processos formativos no âmbito de atividades de extensão. Até o momento foram oferecidas duas oficinas. Uma delas replicando o método de análises de simetrias dirigido aos ladrilhos hidráulicos. Outra, introduzindo o mesmo tipo de análise dirigido ao estudo de artefatos de ferro frequentes na arquitetura dos casarões pelotenses. Desta maneira, relatam-se aqui os resultados obtidos, buscando-se contribuir à atividade docente de representação gráfica digital. Considera-se que tais relatos exemplificam a possibilidade de um trabalho interdisciplinar: geometria, projeto e patrimônio arquitetônico, desde um primeiro momento de formação.

\section{Metodologia}

Para a estruturação de cada oficina foram então abordados conceitos geométricos que pudessem sustentar as atividades de análise dos elementos do patrimônio em questão. O conceito de simetria, já identificado para o estudo dos ladrilhos hidráulicos. E, para o estudo dos artefatos em ferro, considerou-se pertinente abordar, além do de simetria, o conceito de recursão.

Em cada caso são identificados os elementos fundamentais das composições selecionadas e suas regras compositivas; $E$, na sequencia, os estudantes devem gerar novos padrões mantendo o mesmo elemento fundamental e alternando as regras das composições.

O desenvolvimento da proposta se deu através das seguintes etapas:

\section{Revisão}

O referencial de Sanz e Moratalla (1999), identifica sete grupos de simetria de friso (figura 1 à esquerda). Estes grupos envolvem transformações de translação (Ta), reflexão em torno de um eixo horizontal (Sr), reflexão em torno de um eixo vertical (Sm), reflexão de eixo horizontal com deslizamento (L) e rotação (Ga).

Para o estudo de recursão foi adotado o referencial de Celani, 2003, o qual sistematiza os tipos de composições sobre este conceito. Nestas, estão envolvidas as regras de substituição, concentricidade (recursão concêntrica), disposição nuclear, simetria cíclica, subdivisão interna e adição, ilustradas à direita da figura 1.

\section{Etapa de seleção dos edifícios e elementos de interesse patrimonial e análise das composições encontradas}

Para o estudo de simetria, foi buscou-se então ampliar o estudo realizado anteriormente, selecionando-se um outro casarão pelotense: o de número 6. Este Casarão está localizado ao redor da Praça Coronel Pedro Osório e está contíguo ao casarão 2, referido anteriormente. Ambos fazem parte de um conjunto tombado pelo IPHAN. A seleção deu-se especialmente pela facilidade de acesso para identificar todos os seus ladrilhos, pois é um edifício de caráter público.

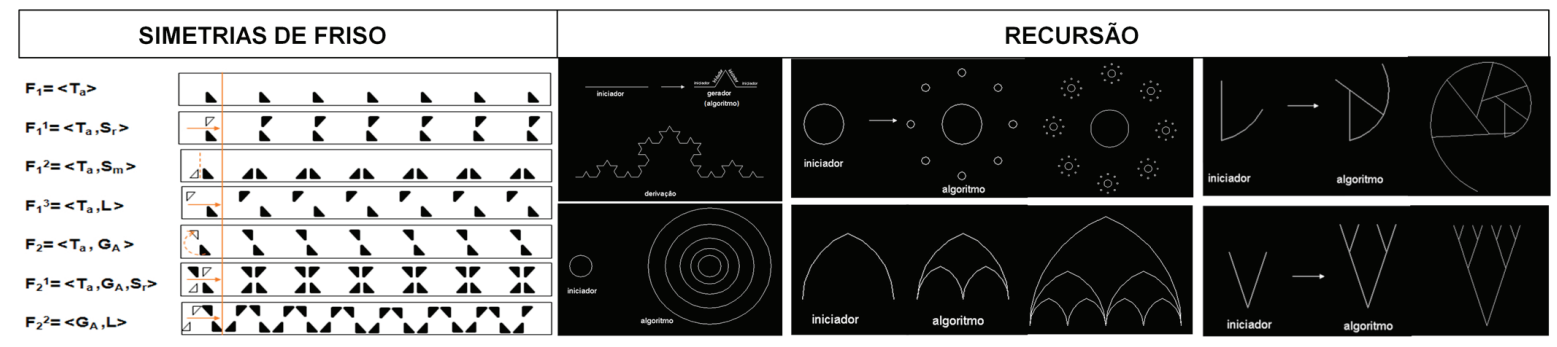

Figura 1: Regras dos Grupos de simetrias de frisos e tipos de recursão. Fonte: Sanz e Moratalla, 1999, e Celani, 2003. 
O Casarão 2, ilustrado na parte superior da figura 2, trata-se de uma construção edificada em 1879. Foi construído por José Izella Merotte e abrigará em breve o Museu da cidade de Pelotas, RS (http://www.pelotas.rs.gov.br/cidade_atracoes/pelotas_atracoes_ casarao6.htm). Em seu interior, foram identificados cinco tipos de ladrilhos que compõem frisos, localizados em pisos e paredes e distribuídos em cinco cômodos, especificados ainda na figura 2.

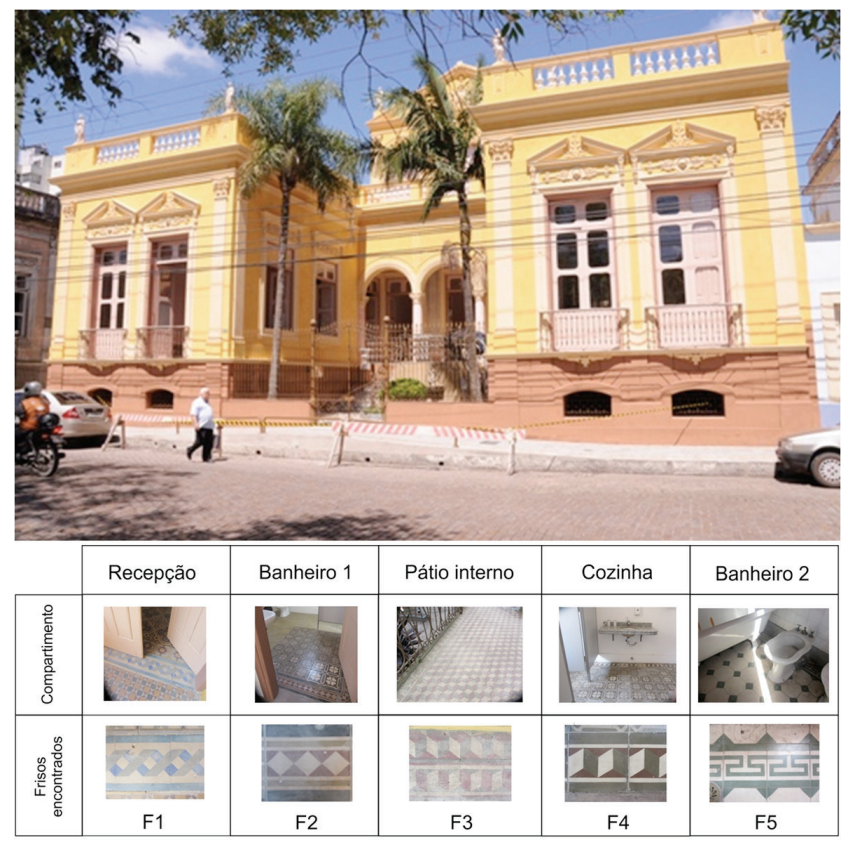

Figura 2: Acima, Casarão 6, Praça Cel. Pedro Osório, Pelotas, RS. Fonte: www.pelotas.rs.gov.br/noticias; Abaixo, tipos de frisos identificados no Casarão 6. Fonte: Autores.

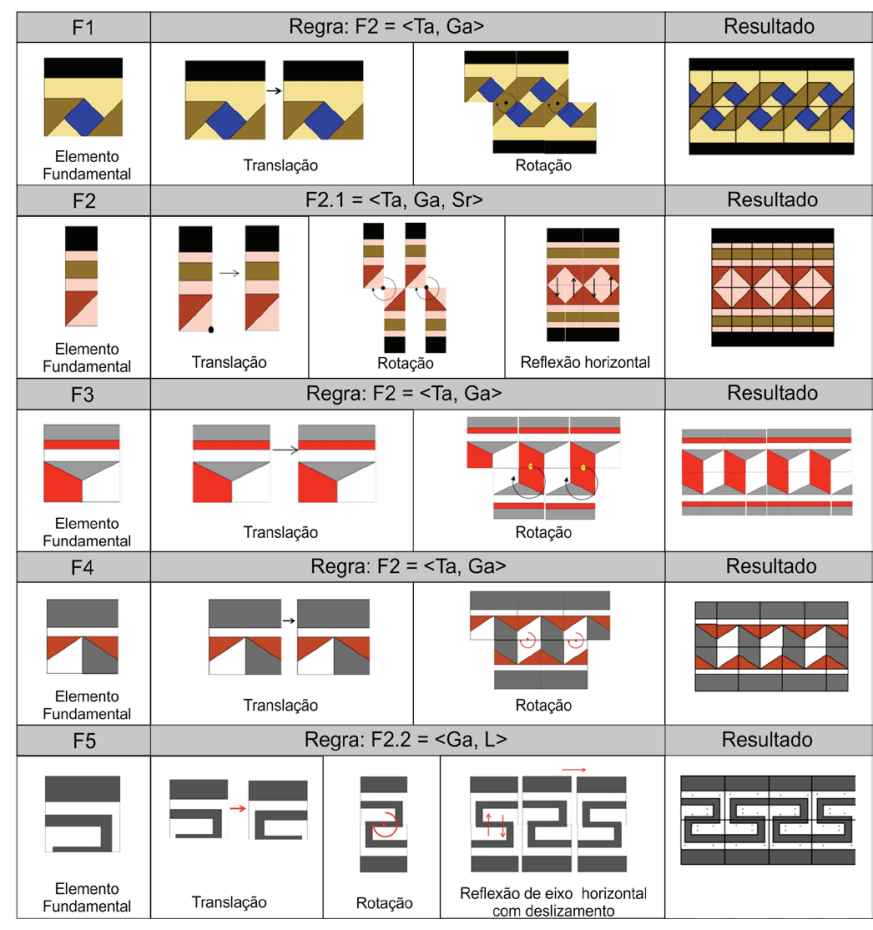

Figura 3: Identificação das regras de simetrias de friso nos ladrilhos hidráulicos do Casarão 6. Fonte: Autores.
Para os cinco frisos identificados foram encontrados três tipos de grupos de simetria, dentre os sete grupos caracterizados em Sanz e Moratalla (1999), conforme especificado na figura 3.

Para o estudo de recursão, foi selecionado um tipo de artefato em ferro: uma janela do tipo bandeira. A bandeira selecionada encontra-se na fachada principal da edificação que abriga a Biblioteca Pública de Pelotas (ilustrada na primeira linha da figura 4), localizada também no entorno da Praça Coronel Pedro Osório.
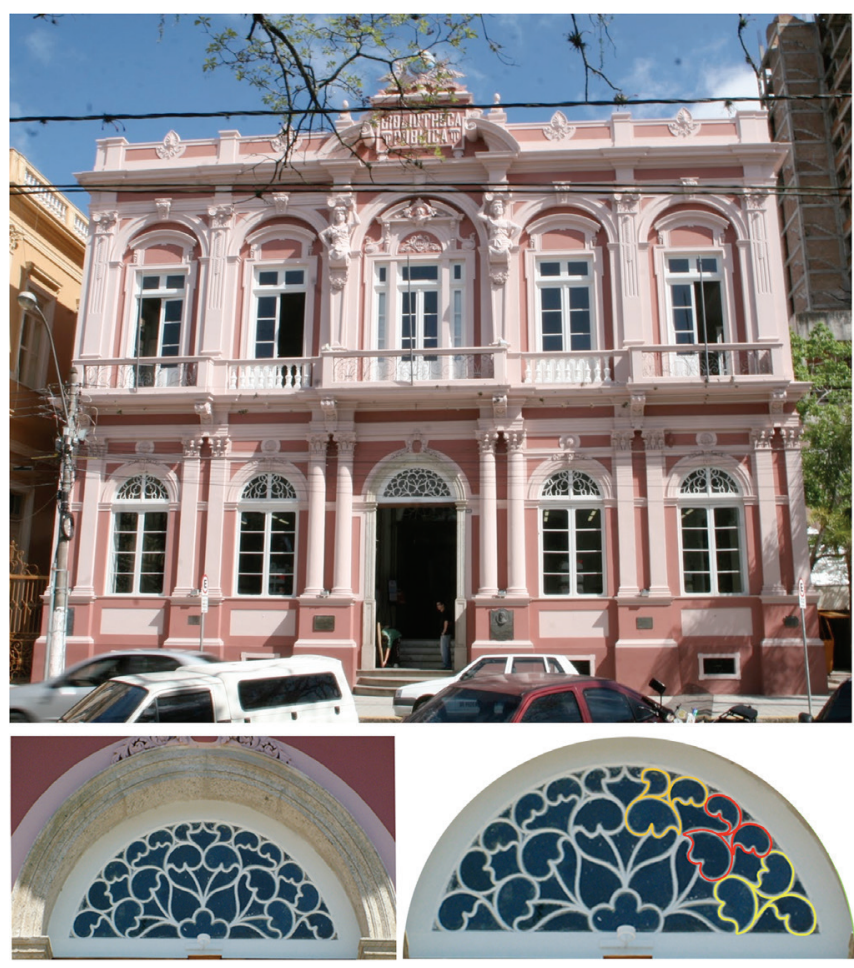

Figura 4: Acima, Biblioteca Pública Pelotense, Praça Coronel Pedro Osório, Pelotas; abaixo, detalhe da esquadria tipo bandeira selecionada para a atividade de análise. Fonte: autores.

Esta edificação foi construída em 1875 e financiada por cavalheiros mais abastados da sociedade local, com o objetivo de colaborar com o conhecimento intelectual e cultural da cidade. Foi também projetada por José Izella Merotte. Alguns materiais para a sua construção foram trazidos da Europa, precisamente de Portugal, tais como os marcos de pedra e o arco de granito de sua entrada principal. Recebeu o acréscimo de um segundo piso entre 1911 e 1913 projetado por Caetano Casaretto. A linguagem formal deste edifício é de influência eclética, com colunas, pilastras e frontão marcando sua fachada, além de balcões e sacadas de púlpito (http://www.pelotas.rs.gov.br/cidade_atracoes/pelotas_ atracoes_biblioteca.htm).

Na parte inferior da figura 4, destaca-se o conjunto de elementos sobre os quais é possível associar um procedimento compositivo a partir do conceito de recursão. Especificamente pode-se caracterizá-lo, sob os tipos listados em Celani (2003) como uma composição por adição de elementos, dispostos em torno de um ponto central. 


\section{Etapa de proposição das atividades didáticas}

Em ambas as oficinas foi proposto que os estudantes gerassem, através de processos informáticos dinâmicos, novas composições por diferentes combinações entre as figuras básicas e as regras identificadas. A tabela ilustrada na figura 5 descreve a proposta para a oficina de simetria.

Foram disponibilizadas as figuras básicas das composições representadas no AutoCad, tanto para as atividades com simetria quanto de recursão. As composições originais dos frisos e da bandeira foram representadas através do processo dinâmico proposto em Celani (2003) (segunda e terceira linha da figura 5). Este permitiu também experimentar a alteração das regras, gerando novas composições.

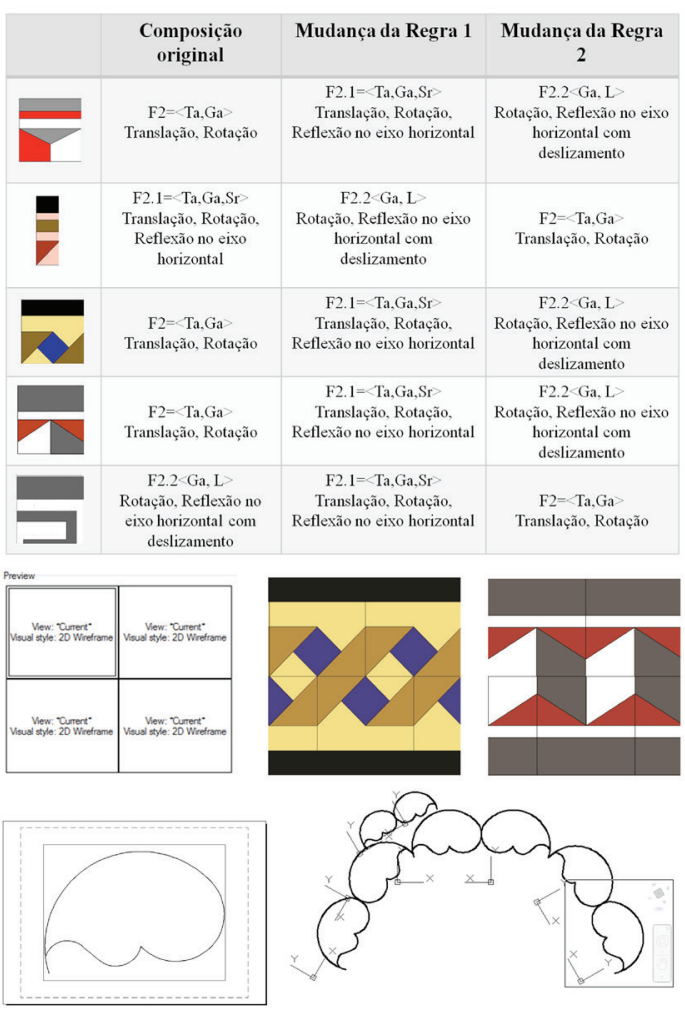

Figura 5: Proposta de exercício para a oficina de simetria; Configuração de janelas de visualização do AUTOCAD e simulações das composições originais dos frisos e da bandeira selecionados para as atividades. Fonte: Autores.

\section{Resultados}

A figura 6 ilustra os resultados das duas oficinas. Nas oito primeiras linhas desta figura estão representadas as composições de frisos. Estas resultam da variação de regras sobre os elementos fundamentais dos frisos anteriormente analisados. Para o elemento fundamental de cada friso foram aplicadas as regras dos grupos F1.1, F2, F2.1 e F2.2 de simetria de frisos. Nas linhas 2, 4 e 8 , estão representadas as composições geradas pela variação do elemento fundamental dos frisos 1,2 e 5 , respectivamente.
Ainda na figura 6 , em sua parte inferior, estão representadas as composições de recursão resultantes da variação sobre o elemento fundamental componente da bandeira analisada. Neste exercício foi considerada a mesma regra de recursão anteriormente identificada no elemento arquitetônico: a esquadria bandeira.

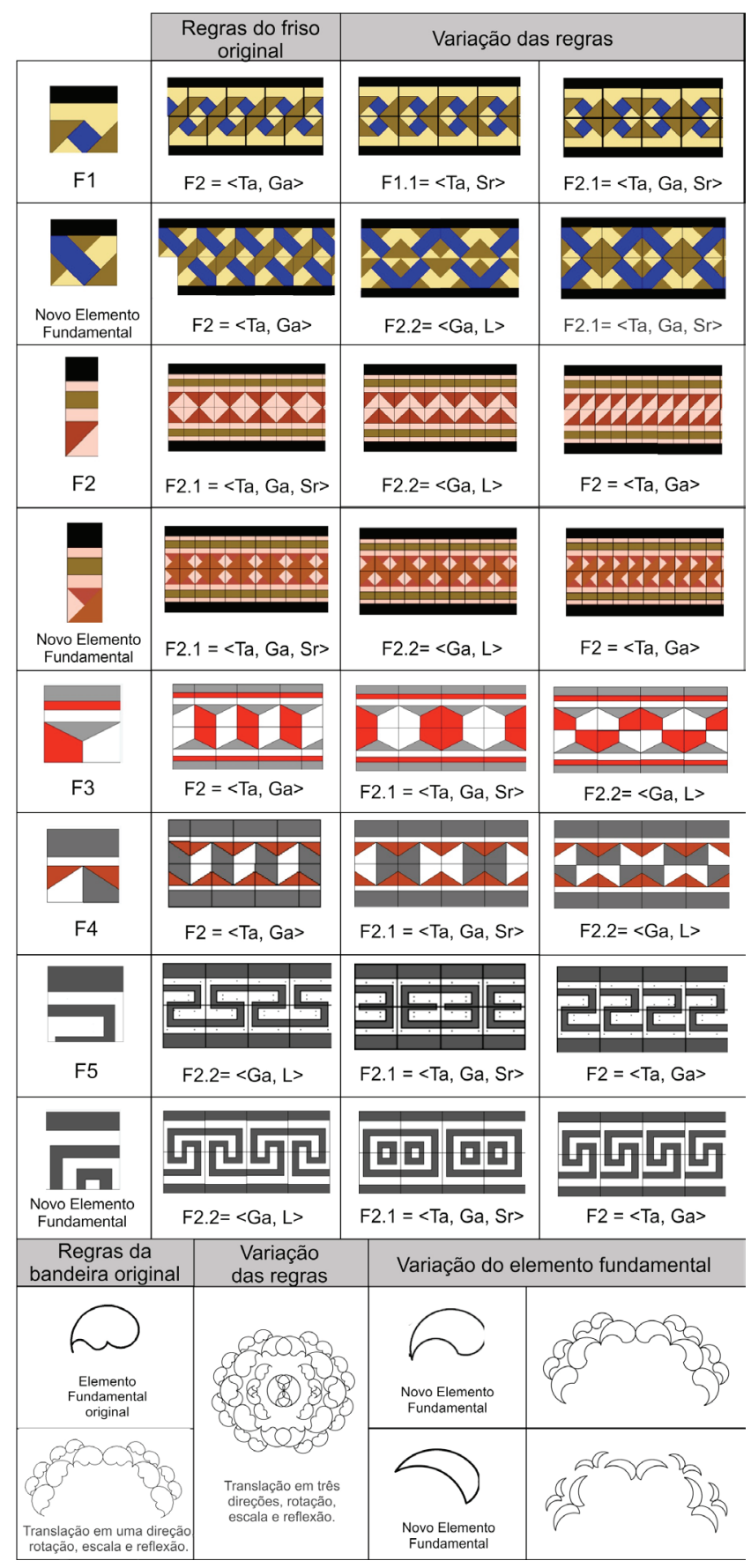

Figura 6: Novas composições de frisos geradas a partir de variação das regras dos grupos de simetria de frisos de Sanz e Moratalla, e do elemento fundamental dos frisos 1,2 e 5 .

Observou-se que a metodologia proposta e o uso de técnicas dinâmicas de representação em exercícios deste tipo tem permitido a melhor compreensão dos princípios compositivos. Isto 
porque exige que o padrão seja desconstruído em seu elemento fundamental e reconstruído a partir de suas regras. E, principalmente, pelo exercício de geração de novos padrões, de maneira automatizada, facilitando a alteração da regra compositiva a partir de um elemento inicial. Através de técnicas tradicionais de representação, por outro lado, era necessário representar cada elemento separadamente a cada etapa de desenho da composição. Este processo talvez estimulasse a pensar primeiro na composição final, sem refletir sobre as regras compositivas envolvidas no processo. Desta maneira, talvez não exploradas para gerar diferentes soluções. 0 processo desenvolvido por técnicas tradicionais de representação não contemplava assim o exercício de reconhecimento das transformações geométricas aplicadas a cada padrão, enfatizando a geração mais intuitiva do projeto final a ser alcançado.

Os resultados têm apontado para: maior compreensão dos conceitos de simetria e recursão, fundamentais no estudo da geometria plana e dos processos compositivos em design; compreensão sobre a estrutura de desenhos complexos, mas os quais são gerados a partir de elementos fundamentais e regras simples; entendimento dos processos envolvidos na geração de cada padrão ou do conjunto deles; conhecimento sobre a arquitetura de interesse patrimonial local; apropriação de técnicas dinâmicas de geração de soluções de projeto.

Considera-se que este exercício de reconhecimento dos princípios compositivos de arquiteturas referenciais, através dos conceitos da geometria plana e processos informáticos dinâmicos de representação, seja fundamental para a ampliação do repertório projetual dos estudantes de arquitetura em fase inicial de formação.

\section{Considerações Finais}

Este trabalho buscou promover atividades de análise e composição de padrões de elementos componentes da arquitetura de interesse patrimonial. Envolveu fundamentalmente o reconhecimento de regras geométricas de simetria e recursão subjacentes à forma destes elementos. Através de técnicas dinâmicas de representação gráfica digital (Celani, 2003) foram desenvolvidas atividades de geração de novos padrões a partir daqueles analisados.

Considera-se que tais tipos de atividade possam estimular a capacidade do estudante de reconhecer ações projetuais, contribuindo para sua percepção dos problemas de projeto, constituindo-se, dessa maneira, em conhecimento para a prática projetual.

\section{Agradecimentos}

Este trabalho se inseriu no âmbito do Projeto PROBARQ (Produção e Compartilhamento de Objetos de Aprendizagem dirigidos ao projeto de arquitetura), o qual buscou estruturar materiais didáticos para os estágios iniciais de formação, a serem disponibilizados de forma irrestrita às comunidades acadêmicas e profissionais da área de arquitetura, em sistemas de acesso na Internet. Agradecemos ao CNPq por financiar o projeto e a PRG/UFPel, pelo apoio dado através de bolsa de ensino, que tornou possível o desenvolvimento desse estudo. Agradecemos também ao Projeto AlfaGaviota/Comunidade Europeia, que possibilitou a realização de oficinas de ensino/aprendizagem em gráfica digital durante o $4^{\circ}$ Workshop da rede, ocorrido na Universidade Federal de Pelotas, em abril de 2013.

\section{Referências}

Borda, A., Pires, J. F., Alves, C. M. (2012) A lógica compositiva de ladrilhos hidráulicos nos casarões de Pelotas. In: IV Congreso Internacional de Expresión Gráfica e IX Congreso Nacional de Profesores de Expresión Gráfica. Gráfica del Diseño:tradición e innovaciones. La Plata: Color Magenta Gráfica, v.01. p.41 - 44

Celani, Gabriela (2003). Cad Criativo. Rio de Janeiro: Editora Campus.

Dametto, A. A. (2009). Os metais no patrimônio arquitetônico urbano de Pelotas, RS - 1870 a 1931. Dissertação. Programa de Pós-graduação em Memória Social e Patrimônio Cultural. Universidade Federal de Pelotas, Brasil.

Léon, Zênia de (1998). PELOTAS, CASARÕES Contam Sua História. São Lourenço do Sul: Editora Hofstatter, 1998, $3^{\circ}$ Vol. 3.

Sanz, M. A. Y Moratalla, A (1999). Simetría. Serie Geometría y Arquitectura II, Cuadernos de Apoyo a la Docencia del Instituto Juan de Herrera. Madri: Publicaciones de la Escuela Superior de Arquitectura de Madrid. 